\title{
Helium Scanning Transmission Ion Microscopy and Electrical Characterization of Glass Nanocapillaries with Reproducible Tip \\ Geometries
}

Ludovit P. Zweifel ${ }^{1}$, Ivan Shorubalko ${ }^{2}$ and Roderick Y.H. Lim ${ }^{1}$

${ }^{1}$ Biozentrum and the Swiss Nanoscience Institute, University of Basel, 4056 Basel, Switzerland

${ }^{2}$ Laboratory for Reliability Science and Technology, EMPA, Swiss Federal Laboratories for Materials Science and Technology, 8600 Dübendorf, Switzerland

\section{Corresponding Author:}

Roderick Y. H. Lim

Biozentrum and the Swiss Nanoscience Institute

University of Basel, Klingelbergstrasse 70

CH-4056 Basel, Switzerland

Phone: +4161267 2083

Fax: +41612672109

E-mail: roderick.lim@unibas.ch 


\section{GNC fabrication parameters and image statistics}

Table S1. Sutter Instruments P-2000 B heat and velocity values with related "heat-on" times and shapes (filament 0, pull 255, delay 170). Heat-on mean and standard deviation values have been obtained from $\mathrm{N}_{1}=8, \mathrm{~N}_{2}=13, \mathrm{~N}_{3}=20, \mathrm{~N}_{4}=11, \mathrm{~N}_{5}=30, \mathrm{~N}_{6}=21$ and $\mathrm{N}_{7}=14$ pulls.

\begin{tabular}{c|cccc}
\hline \multicolumn{1}{c}{ Program } & Heat & Velocity & Heat-on [s] & Shape \\
\hline $\mathbf{1}$ & 460 & 25 & $0.92 \pm 0.03$ & Bullet \\
$\mathbf{2}$ & 460 & 18 & $0.94 \pm 0.03$ & Bullet \\
$\mathbf{3}$ & 600 & 15 & $0.59 \pm 0.02$ & Blunt \\
$\mathbf{4}$ & 400 & 30 & $1.32 \pm 0.05$ & Blunt \\
$\mathbf{5}$ & 400 & 25 & $1.30 \pm 0.04$ & Hour-glass \\
$\mathbf{6}$ & 400 & 20 & $1.27 \pm 0.05$ & Hour-glass \\
& 300 & 40 & $4.31 \pm 0.35$ & Hour-glass \\
\hline
\end{tabular}

Table S2. Reproducibility of GNCs determined by HeSTIM image analysis. Out of 119 GNCs (fabricated using programs 1 to 7), 85 GNCs (“\# of imaged GNCs”) were successfully imaged. From these, 65 GNCs (“\# of good GNCs") revealed the expected structure to give an average reproducibility of $79 \%$.

\begin{tabular}{|l|c|c|c|c|c|c|c|}
\hline Program & 1 Bullet & 2 Bullet & 3 Blunt & 4 Blunt & 5 Hourglass & 6 Hourglass & 7 Hourglass \\
\hline $\begin{array}{l}\text { \# of imaged } \\
\text { GNCs }\end{array}$ & 14 & 11 & 11 & 12 & 12 & 11 & 14 \\
\hline $\begin{array}{l}\text { \# of "good" } \\
\text { GNCs }\end{array}$ & 10 & 10 & 10 & 10 & 7 & 8 & 10 \\
\hline $\begin{array}{l}\% \\
\text { reproducibility }\end{array}$ & 71 & 91 & 91 & 83 & 58 & 87 & 71 \\
\hline
\end{tabular}




\section{Dependence of longitudinal intensity profile on the altitudinal angle}

Imaging an individual GNC at different altitudinal angles (angle between the horizontal plane and the GNC axis) reveals the linear dependence of intensity on the thickness of the material which He ions have to pass.
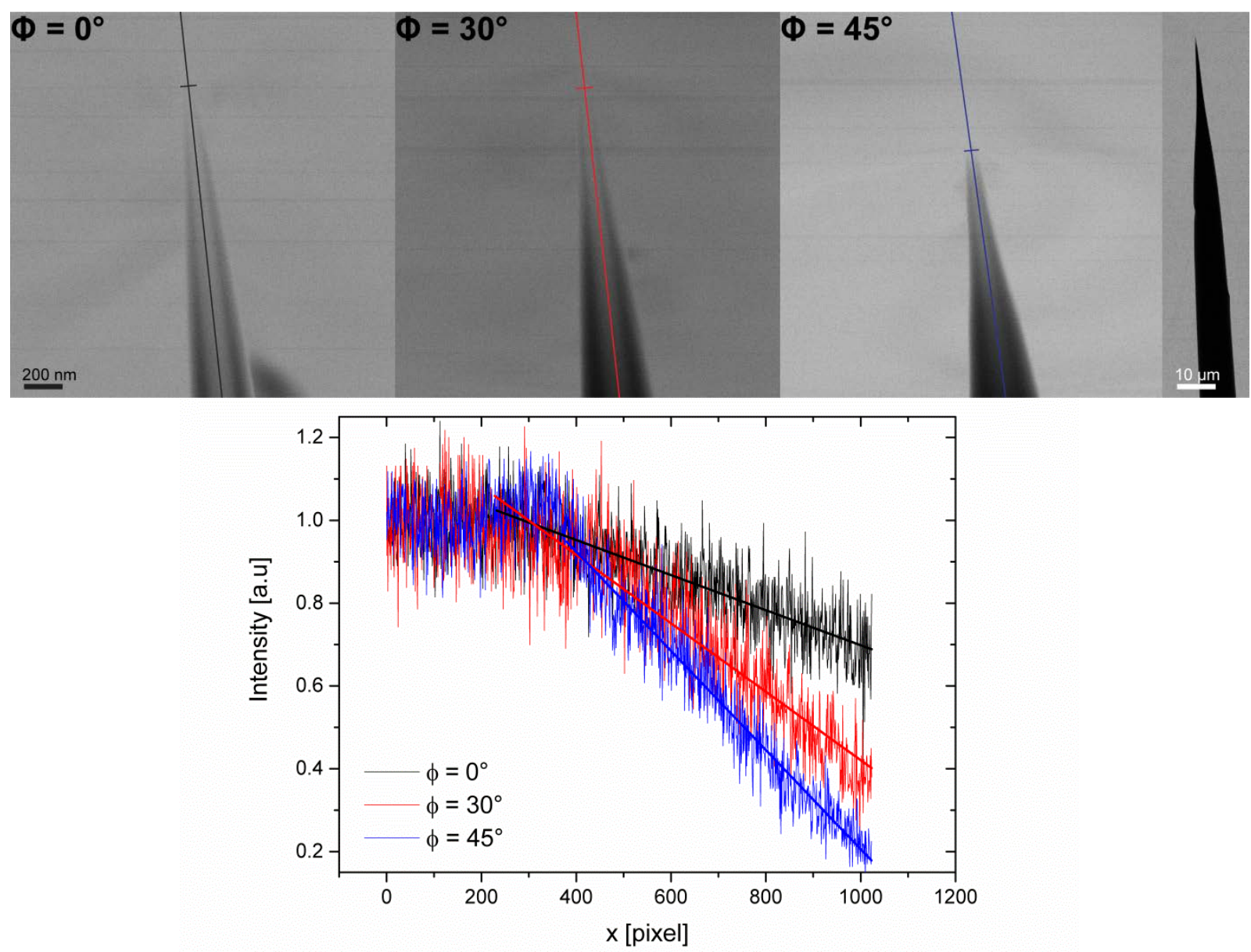

Figure S1. The same GNC imaged under different altitudinal angles and corresponding longitudinal intensity profiles $(\mathrm{FOV}=2 \times 2 \mu \mathrm{m}$ and $1024 \times 1024$ pixels, scale bar $=200 \mathrm{~nm})$. Longitudinal profiles are plotted along the prolonged GNC axes as indicated. The linear profiles correspond to the range between tip openings (depicted with a small perpendicular bar) to the bottom of the images. The rightmost image is an overview $(\mathrm{FOV}=100 \times 100 \mu \mathrm{m}$ and $1024 \times 1024$ pixels of which an area of 206x911 pixels is shown, scale bar $=10 \mu \mathrm{m})$. 


\section{GNC image gallery}

The following images show the GNCs that were fabricated from each respective program as summarized in Table S1. For each program $2 \times 2$ binned zoom-ins of up to ten different GNCs $(\mathrm{FOV}=2 \times 2 \mu \mathrm{m}$ and $1024 \times 1024$ pixels, scale bar $=200 \mathrm{~nm})$ and a representative overview $(\mathrm{FOV}=100 \times 100 \mu \mathrm{m}$ and $1024 \times 1024$ pixels of which an area of 206x911 pixels is shown, scale bar $=10 \mu \mathrm{m}$ ) are shown.

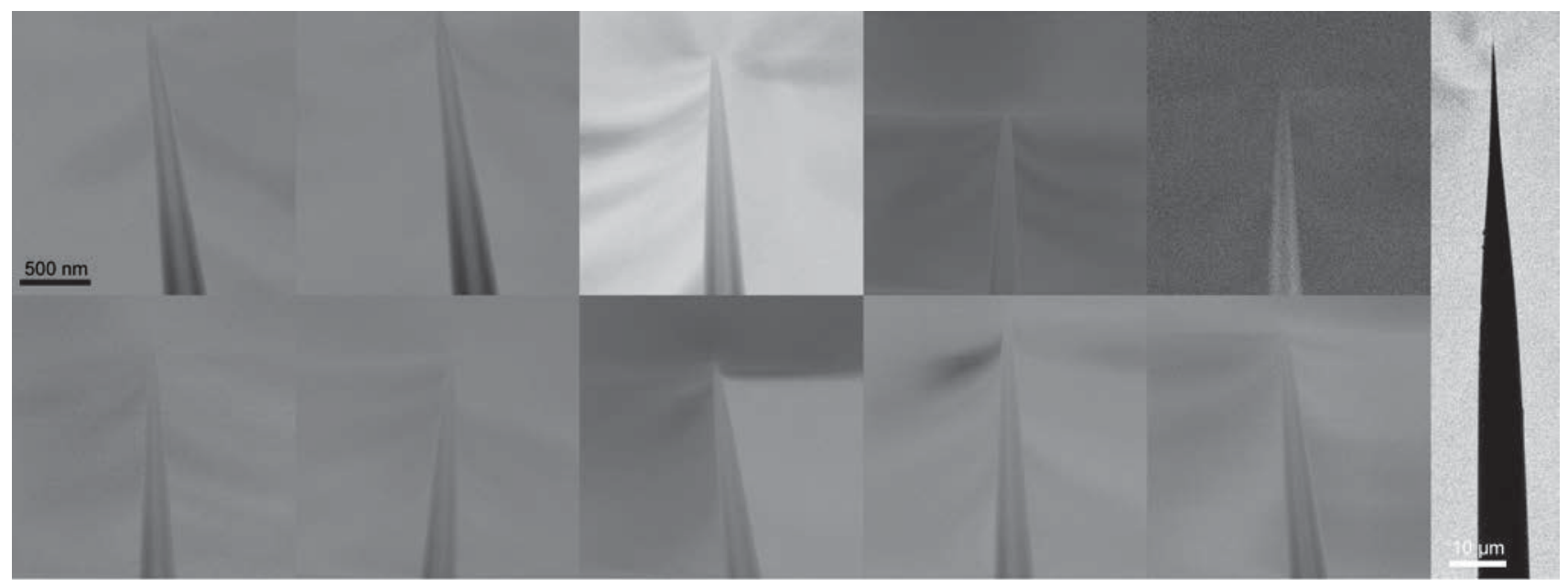

Figure S2. Bullet-like tips from program 1

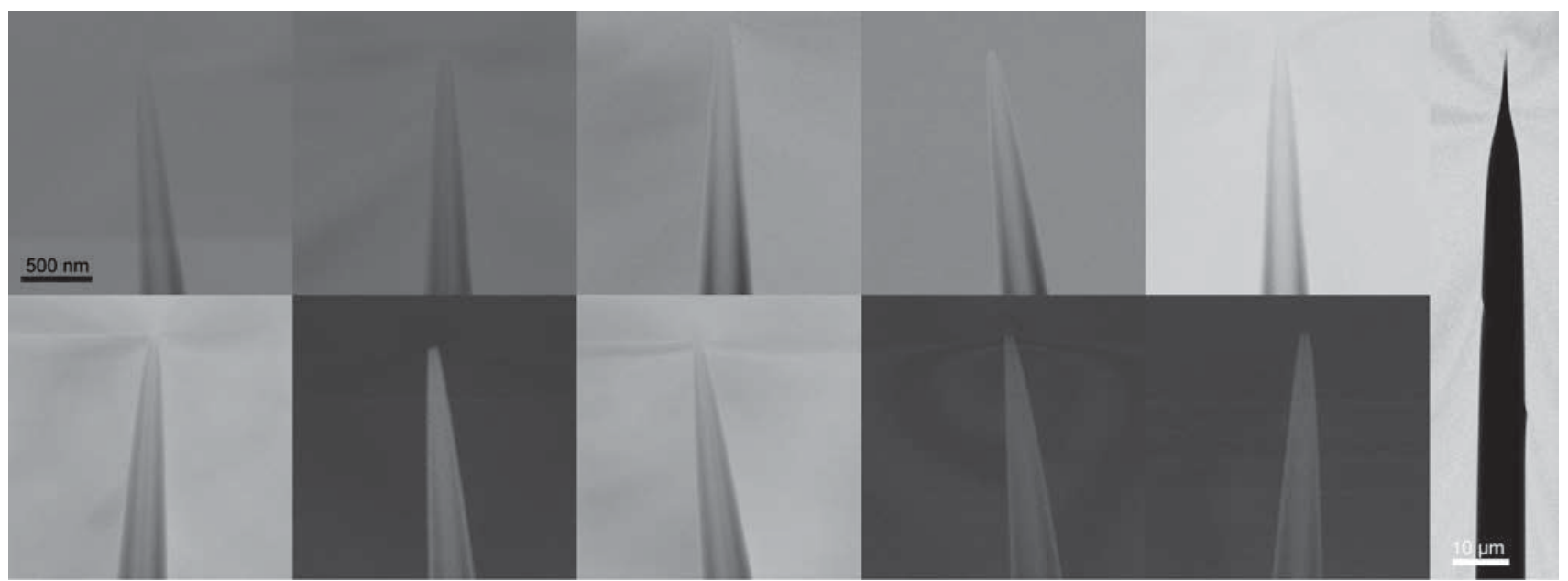

Figure S3. Bullet-like tips from program 2 


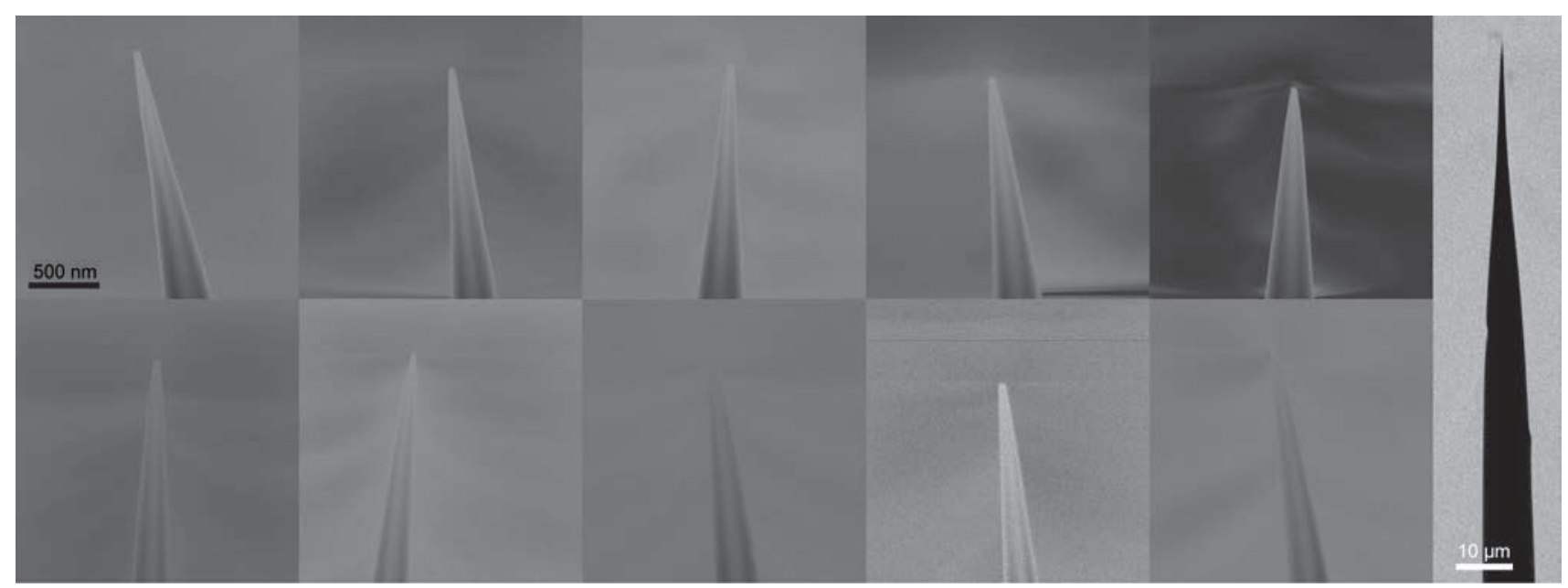

Figure S4. Blunt-ended tips from program 3

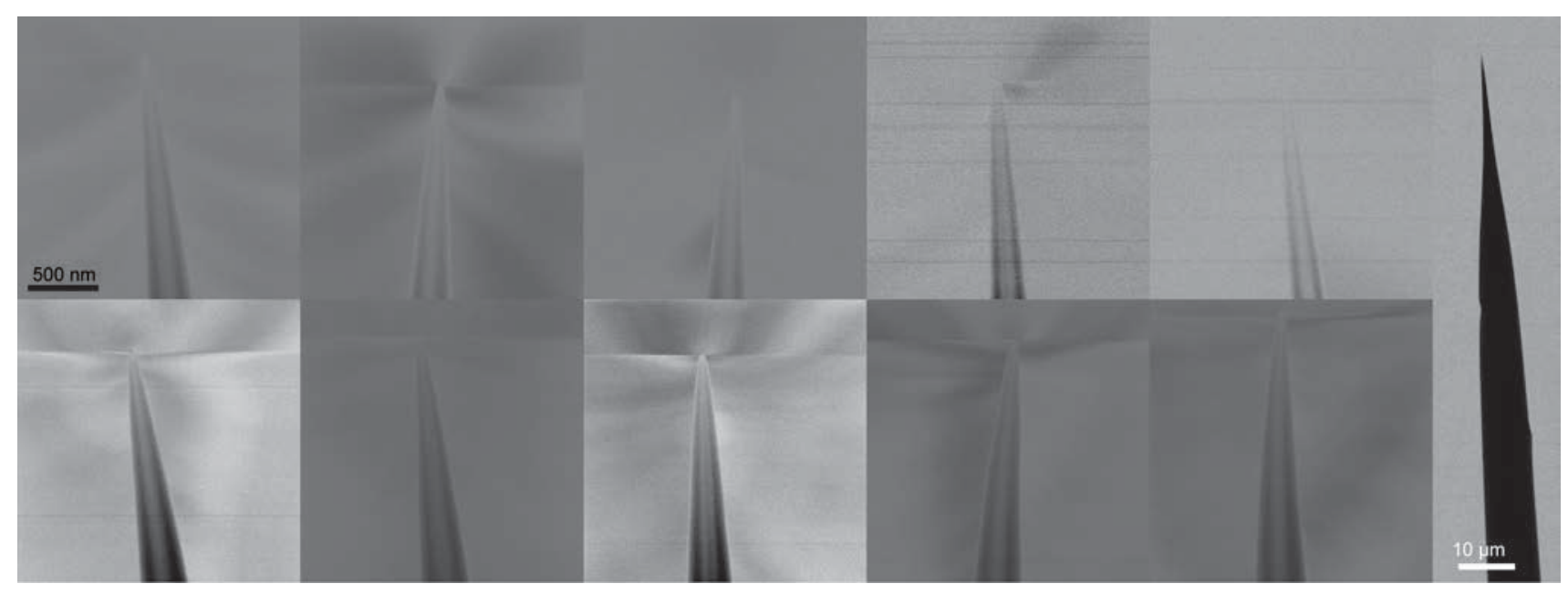

Figure S5. Blunt-ended tips from program 4

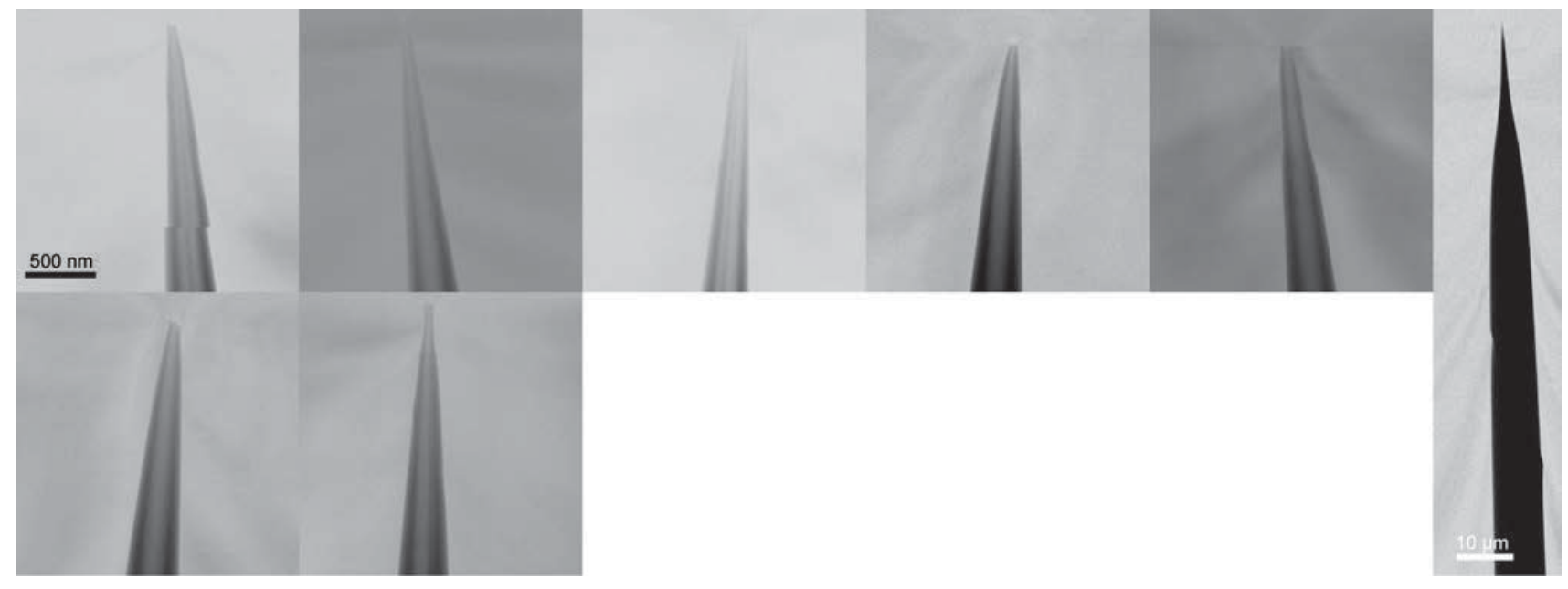

Figure S6. Hourglass tips from program 5 


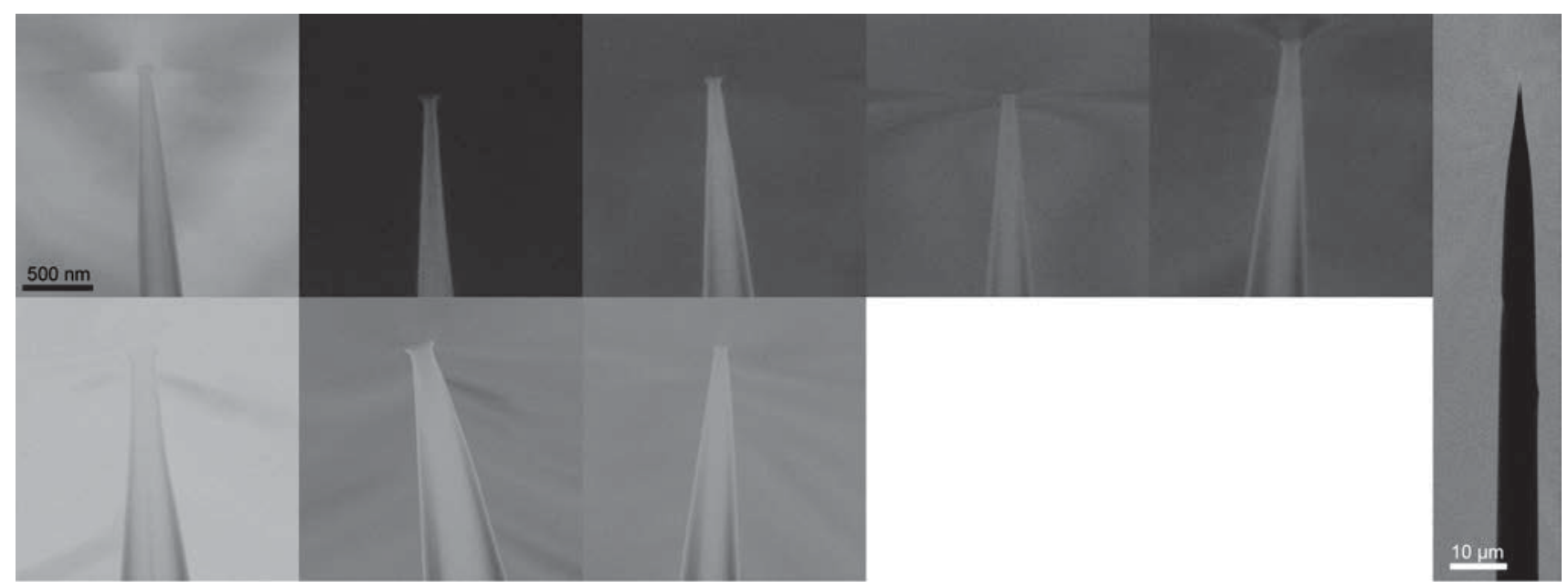

Figure S7. Hourglass tips from program 6

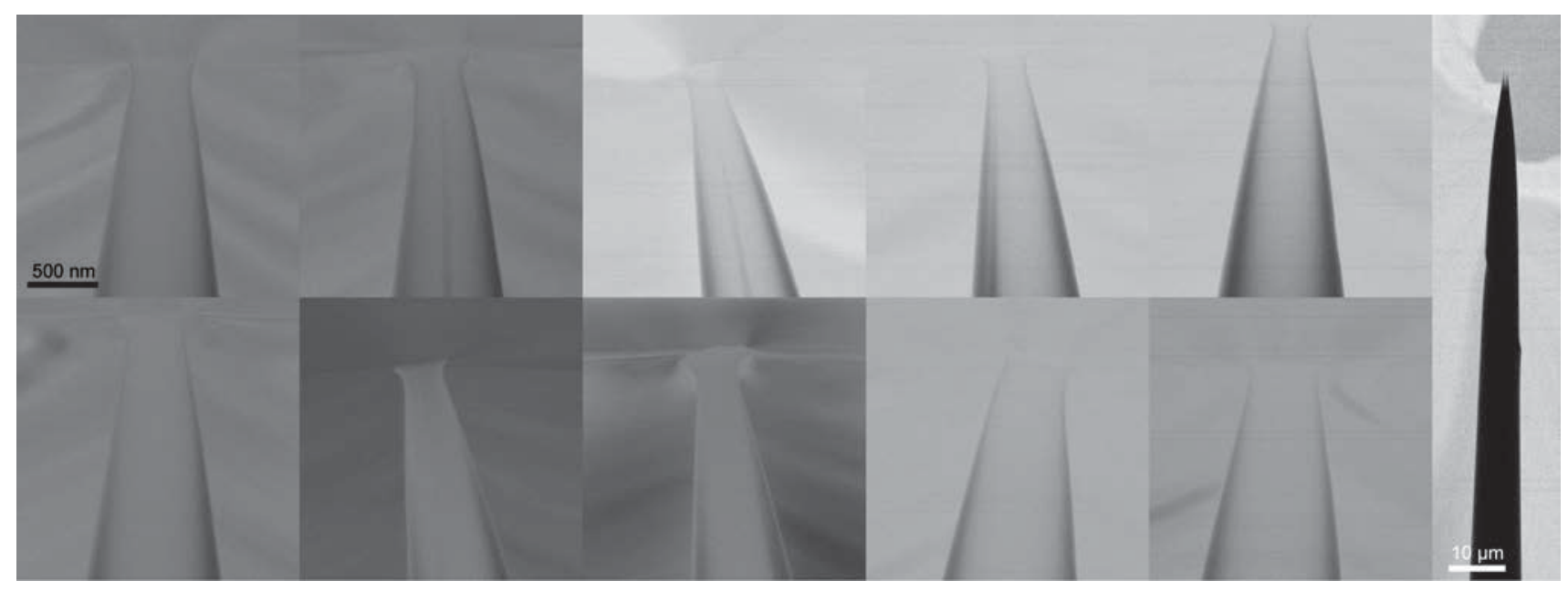

Figure S8. Hourglass tips from program 7 


\section{Current to voltage curve}

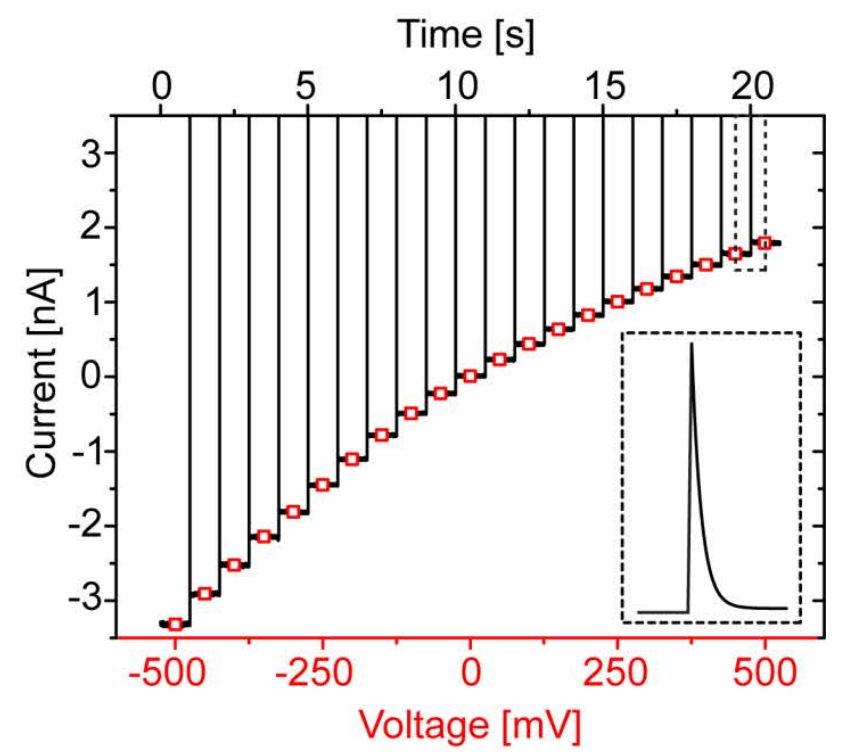

Figure S9. A typical current to voltage response for a bullet-like GNC (red open boxes). The black line is the corresponding contiguous time to current trace, where the applied voltage is stepwise increased $(+50 \mathrm{mV})$ after every second, starting from $-500 \mathrm{mV}$. Dashed box and inset: Each voltage step will initially $(\mathrm{t} \rightarrow 0$ ) drop entirely over the reservoirs, which leads to the current spikes at the beginning of each step. The current will then decay according to the charging of the capacitance between the solution and GNC wall before it stabilizes at a value solely defined by the pore resistance. Current mean values at a given voltage (red open boxes) are therefore obtained from averaging over stable current-time traces $(\mathrm{t}$ $>0.2 \mathrm{~s})$ after each voltage step. 


\section{BSA translocation through GNCs: Normalized current drop and event duration distributions}

Normalized current drop and event duration values in the main text are mean values over the entire distribution of events. For ease of comparison with literature values, ${ }^{1,2}$ distributions of event duration and normalized current drops are plotted with log-normal (Fig. S10) and normal fits (Fig. S11), respectively. See also Tables S3 and S4.

A

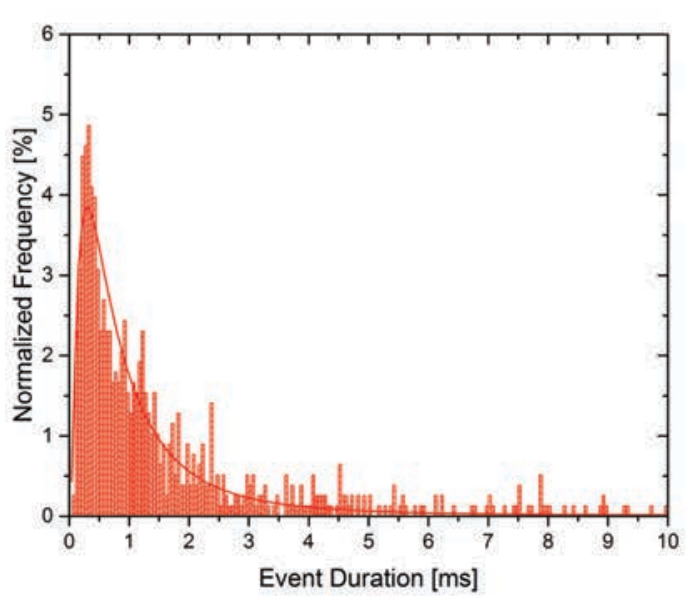

C

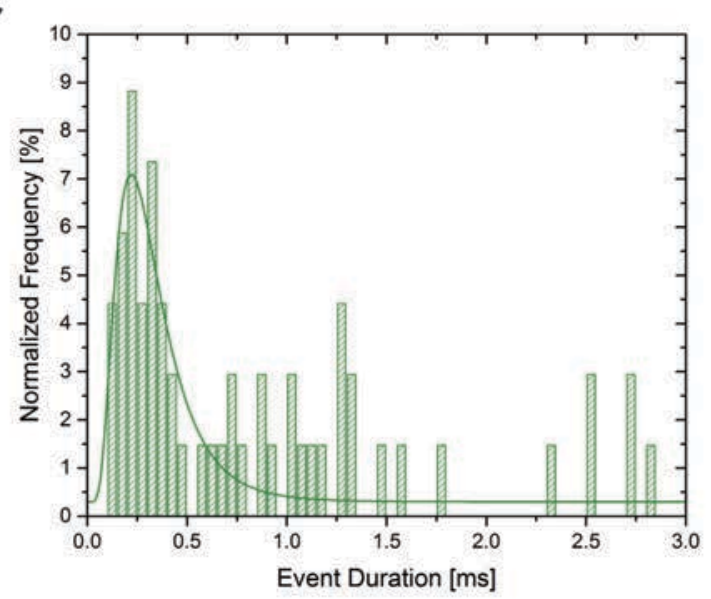

B

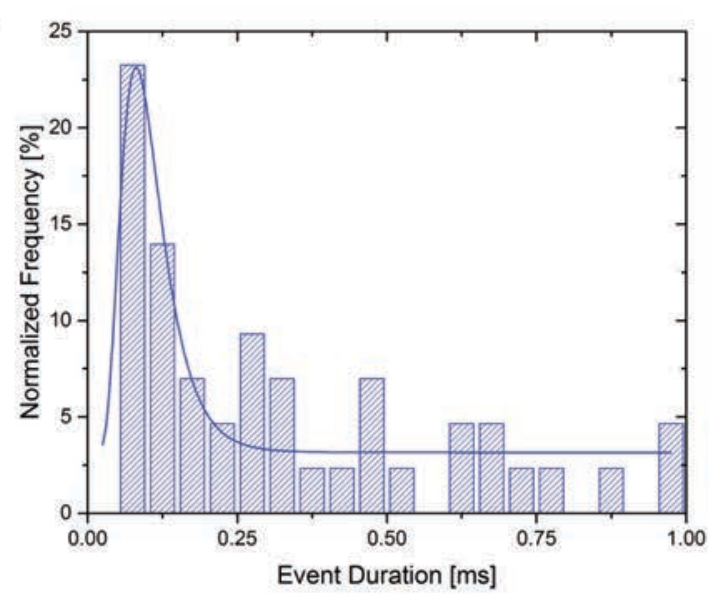

D

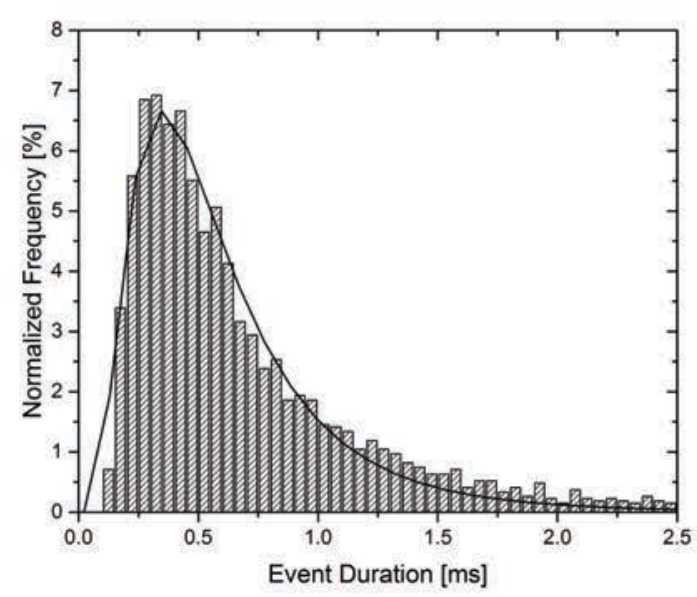

Figure S10. Event duration histograms (binning) and corresponding log-normal fits, with (A) Program 3 (blunt $d_{P}=25 \mathrm{~nm}$ and $\left.\theta=4^{\circ}\right)(\mathbf{B})$ Program 2 (bullet $d_{P}=39 \mathrm{~nm}$ and $\theta=5.6^{\circ}$ ) $\left(\mathbf{C}\right.$ ) Program 4 (blunt $d_{P}=$ $30 \mathrm{~nm}$ and $\theta=5^{\circ}$ ) and (D) Program 5 (hourglass $d_{P}=43 \mathrm{~nm}$ and $\theta=4.4^{\circ}$ ). The obtained fit parameters are summarized in Table S3. 
A

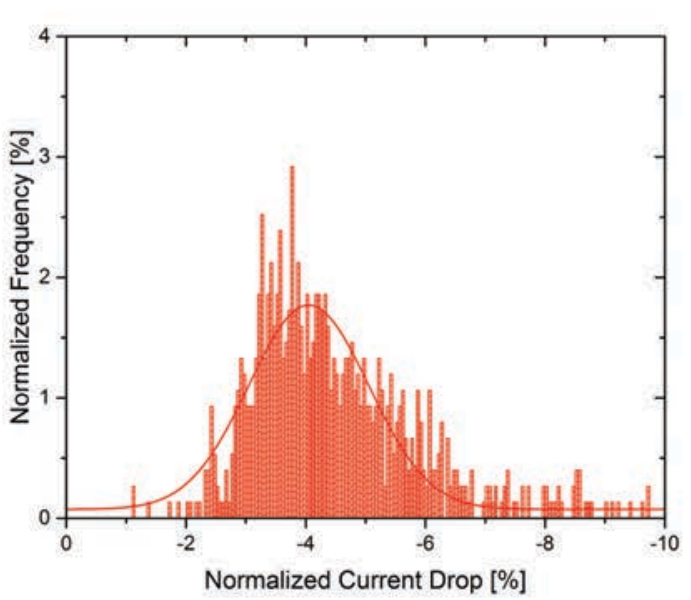

C

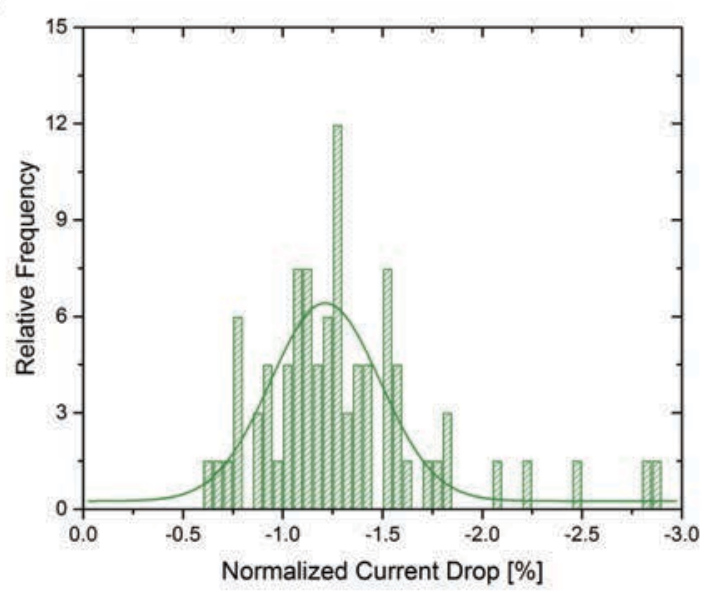

B

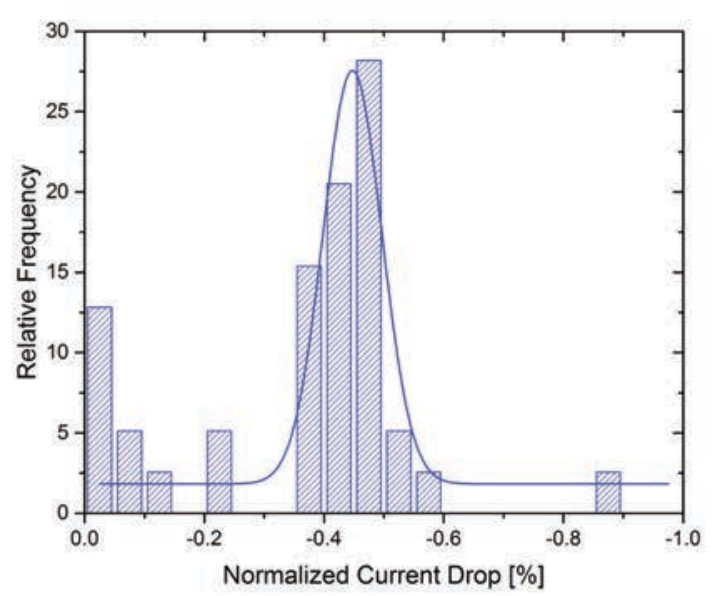

D

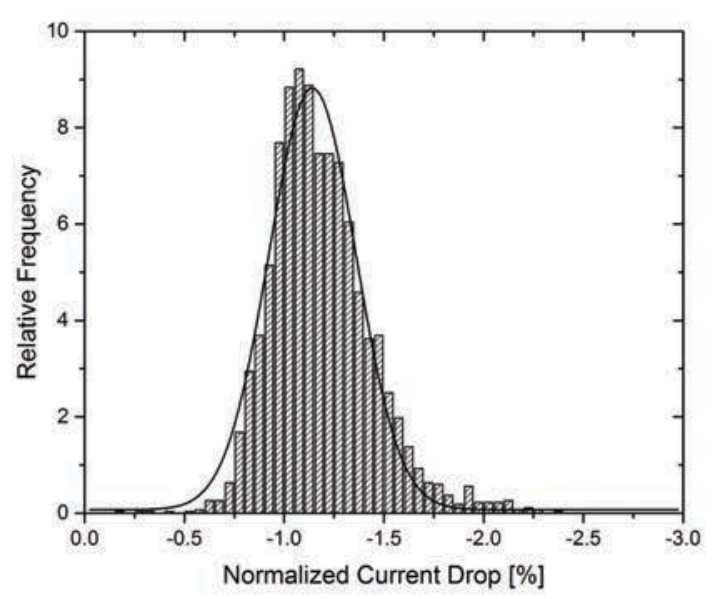

Figure S11. Normalized current drop histograms and corresponding normal fits of the scatter plots shown in the main text (Fig. 5 C), with (A) Program 3 (blunt $d_{P}=25 \mathrm{~nm}$ and $\theta=4^{\circ}$ ) (B) Program 2 (bullet $d_{P}=$ $39 \mathrm{~nm}$ and $\left.\theta=5.6^{\circ}\right)(\mathbf{C})$ Program 4 (blunt $d_{P}=30 \mathrm{~nm}$ and $\theta=5^{\circ}$ ) and (D) Program 5 (hourglass $d_{P}=43$ $\mathrm{nm}$ and $\theta=4.4^{\circ}$ ). The obtained fit parameters are summarized in Table S3. 
Table S3. Event duration and mean current drops from the histograms in Fig. S10 and S11 obtained for BSA translocation in PBS $150 \mathrm{mM} \mathrm{NaCl} \mathrm{pH} 7.2(10 \mathrm{kHz}$ cut-off at 500mV).

\begin{tabular}{|c|c|c|}
\hline Pulling parameter & Event duration & Current drop \\
\hline $\begin{array}{c}\text { Program 3 } \\
\text { (Fig. S10A and Fig. S11A) }\end{array}$ & $755 \pm 12 \mu \mathrm{s}$ & $24.7 \pm 4.7 \mathrm{pA}$ \\
\hline $\begin{array}{c}\text { Program 2 } \\
\text { (Fig. S10B and Fig. S11B) }\end{array}$ & $96 \pm 8 \mu \mathrm{s}$ & $10.2 \pm 1.1 \mathrm{pA}$ \\
\hline $\begin{array}{c}\text { Program 4 } \\
\text { (Fig. S10C and Fig. S11C) }\end{array}$ & $290 \pm 14 \mu \mathrm{s}$ & $18.5 \pm 9.3 \mathrm{pA}$ \\
\hline $\begin{array}{c}\text { Program 5 } \\
\text { (Fig. S10D and Fig. S11D) }\end{array}$ & $506 \pm 13 \mu \mathrm{s}$ & $23 \pm 5.8 \mathrm{pA}$ \\
\hline
\end{tabular}

Table S4. Literature values for BSA translocation through GNCs. Ref. [1] refers to mean current drops and ref. [2] reports the mean of the blockage amplitude distribution. Both references used 1x TE, $1 \mathrm{M}$ $\mathrm{KCl} \mathrm{pH} 8(10 \mathrm{kHz}$ cut-off at 500mV).

\begin{tabular}{|l|l|l|}
\hline \multicolumn{1}{|c|}{ Pulling parameter } & Event durations & \multicolumn{1}{c|}{ Current drop } \\
\hline $\begin{array}{l}\mathrm{f}=0, \mathrm{p}=225, \mathrm{~d}=170, \\
\mathrm{~h}=600 \mathrm{v}=25[1]\end{array}$ & $\sim 50-\sim 200 \mu \mathrm{s}$ & $\begin{array}{l}\sim 10-\sim 100 \mathrm{pA} \\
\text { mean current drop }\end{array}$ \\
\hline $\begin{array}{l}\mathrm{f}=0, \mathrm{p}=200, \mathrm{~d}=140, \\
\mathrm{~h}=620 \mathrm{v}=30[2]\end{array}$ & $\sim 180-\sim 600 \mu \mathrm{s}$ & $\begin{array}{l}\sim 215 \mathrm{pA} \text { mean } \\
\text { peak to peak }\end{array}$ \\
\hline
\end{tabular}




\section{HeSTIM resolution}

All GNCs were imaged at a resolution of $0.95 \mathrm{~nm}^{2}$ per pixel $(\mathrm{FOV}=2 \mu \mathrm{m}$ and $2048 \mathrm{x} 2048$ pixels). Representative GNCs from programs 1 (bullet), 3 (blunt) and 5 (hourglass) exhibiting the smallest pore sizes are shown in Figures S12 - S14.
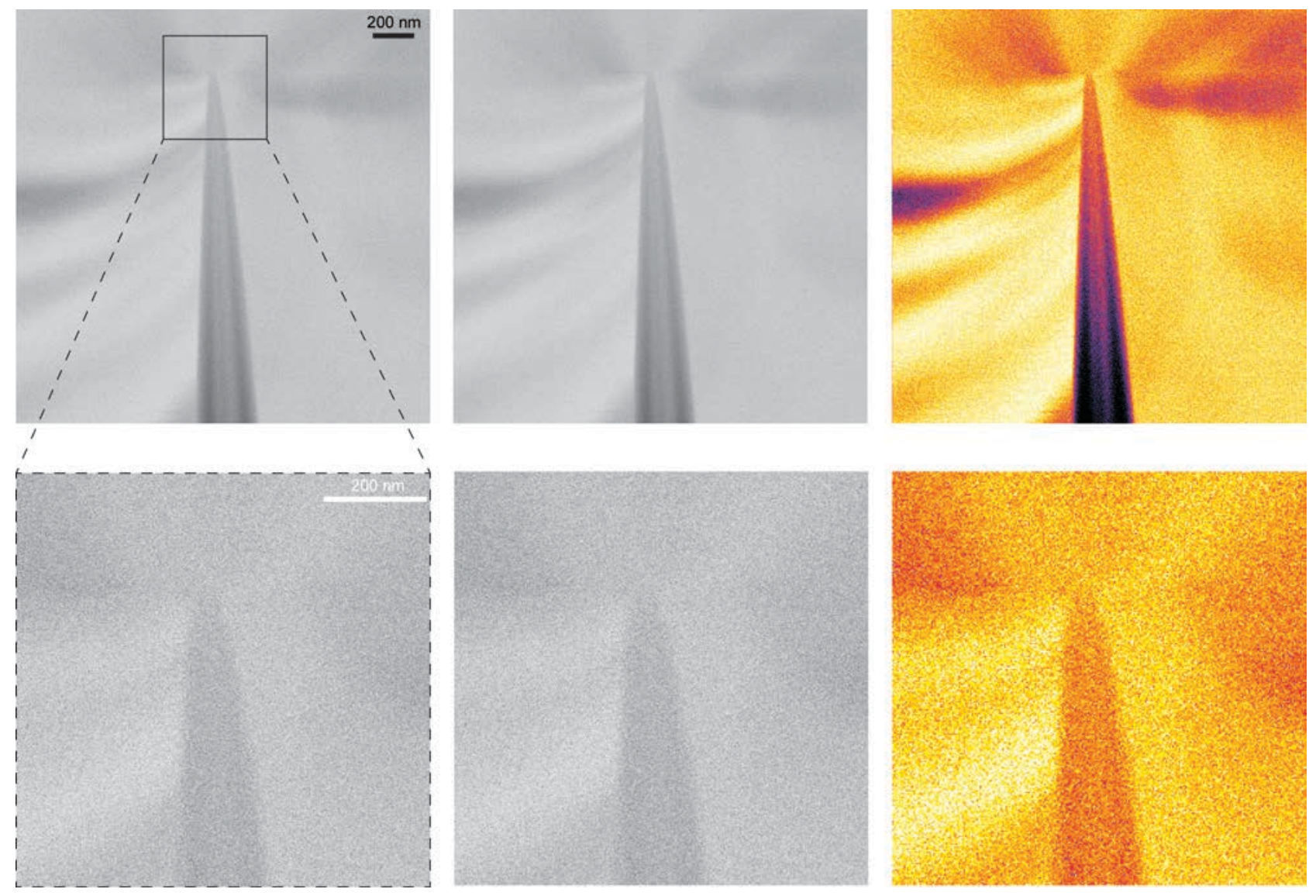

Figure S12. Representative bullet-like GNC from program 1. (Upper row) The unmodified original image (left) was obtained with a FOV $=2 \times 2 \mu \mathrm{m}$ and resolved at 2048x2048 pixels. This has been contrast enhanced and $2 \times 2$ binned (middle and right) for improved clarity. (Lower row) Corresponding zoom-ins provide a $\mathrm{FOV}=500 \times 500 \mathrm{~nm}$ resolved at $512 \times 512$ pixels. 

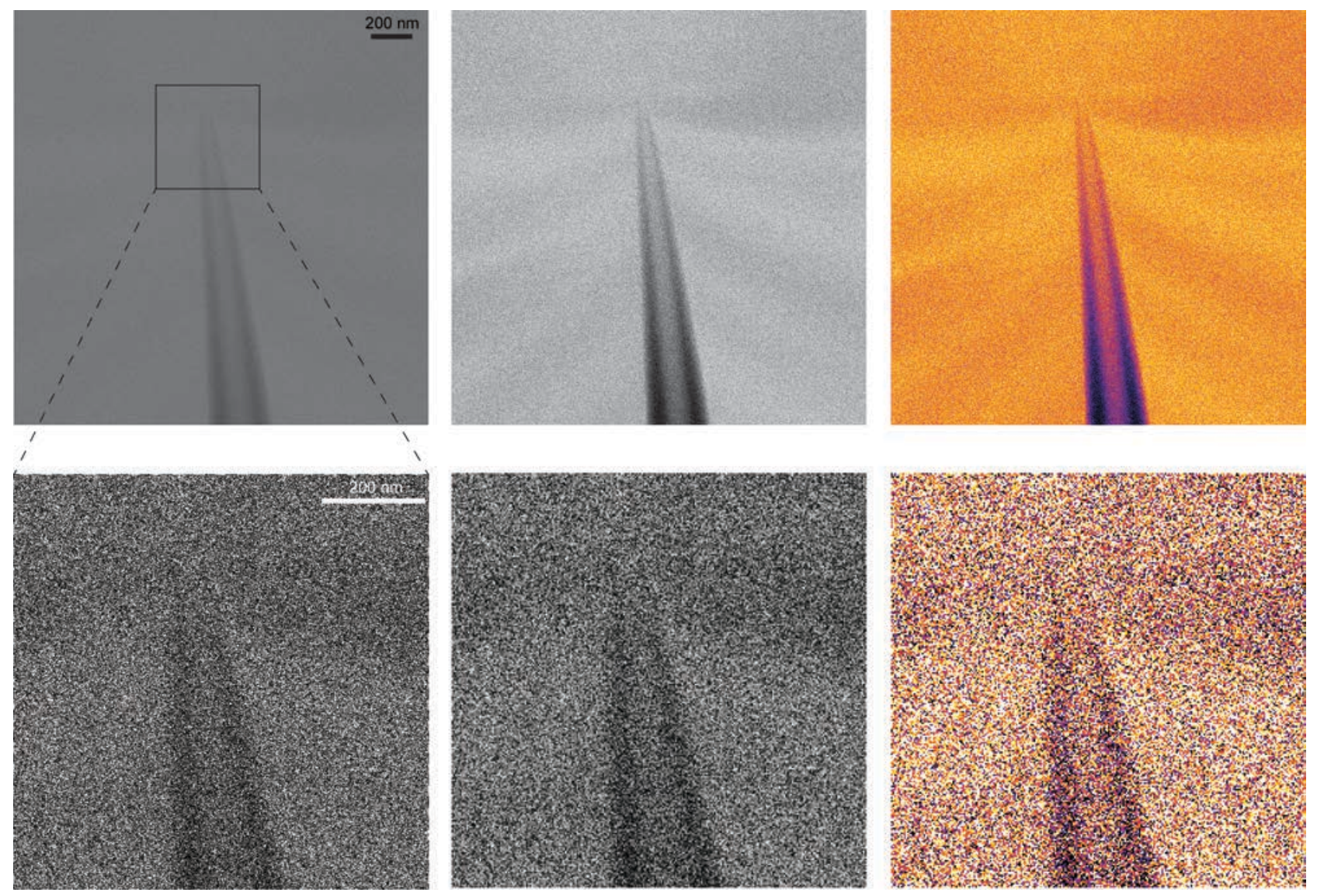

Figure S13. Representative blunt-ended GNC from program 3. (Upper row) The unmodified original image (left) was obtained with a FOV $=2 \times 2 \mu \mathrm{m}$ and resolved at $2048 \times 2048$ pixels. This has been contrast enhanced and 2x2 binned (middle and right) for improved clarity. (Lower row) Corresponding zoom-ins provide a $F O V=500 \times 500 \mathrm{~nm}$ resolved at $512 \times 512$ pixels. 

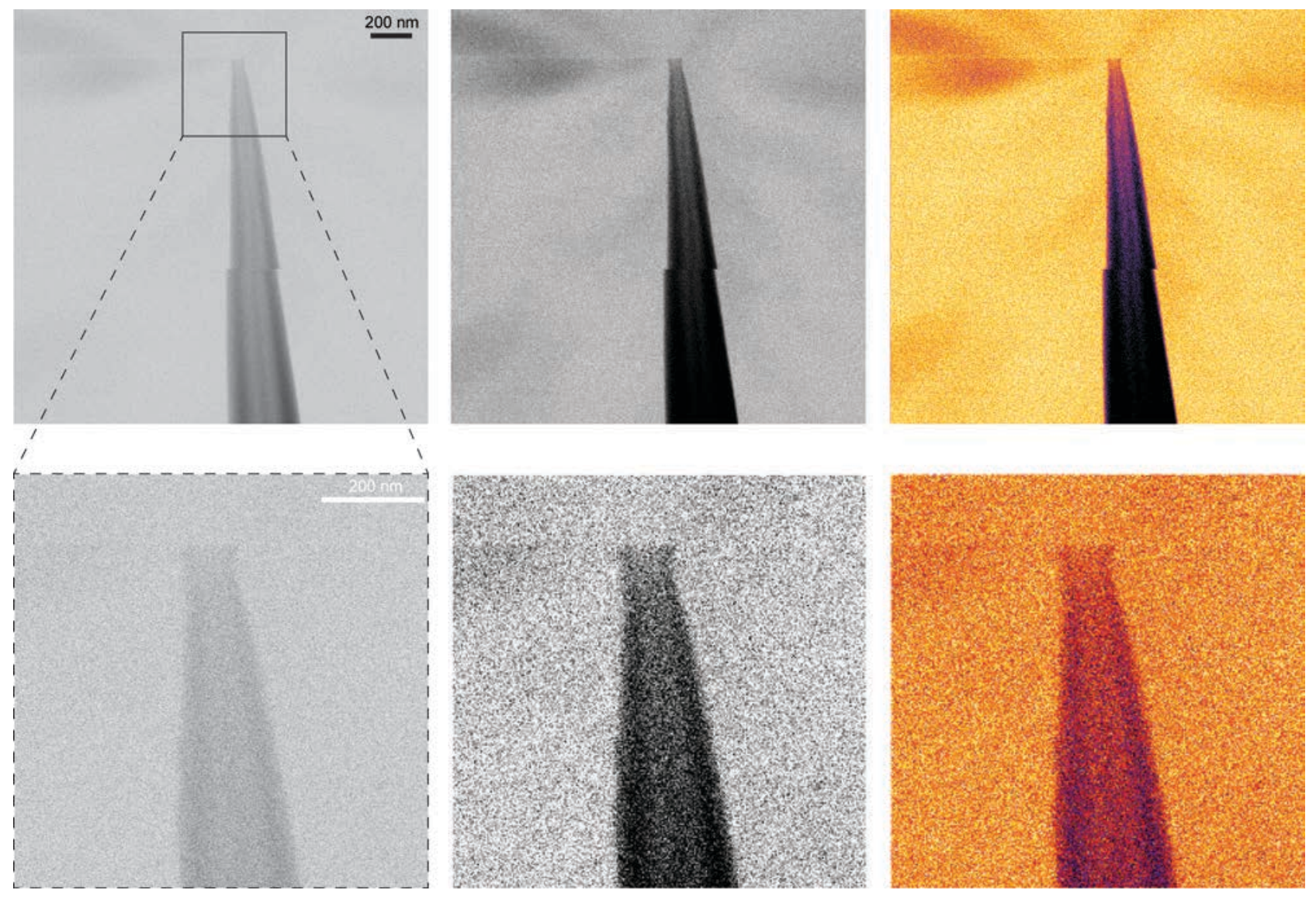

Figure S14. Representative hourglass-shaped GNC from program 5. (Upper row) The unmodified original image (left) was obtained with a FOV $=2 \times 2 \mu \mathrm{m}$ and resolved at 2048x2048 pixels. This has been contrast enhanced and $2 \times 2$ binned (middle and right) for improved clarity. (Lower row) Corresponding zoom-ins provide a $\mathrm{FOV}=500 \times 500 \mathrm{~nm}$ resolved at $512 \times 512$ pixels. 


\section{Influence of plasma cleaning}

Plasma cleaning was found to impact the $I$ - $V$ characteristics of the GNCs to varying extents depending on plasma power and exposure time. However, a $10 \mathrm{~min}$ treatment of $\mathrm{O}_{2}$-plasma at a power of $12.5 \mathrm{~W}$ and 0.3 mbar showed negligible effects. The same setting was subsequently applied to hydrophilize each GNC to promote wetting. A 3 min air plasma at $10 \mathrm{~W}$ and 0.8 mbar also provided a moderate cleaning procedure for GNCs prior to HeSTIM imaging. 
A

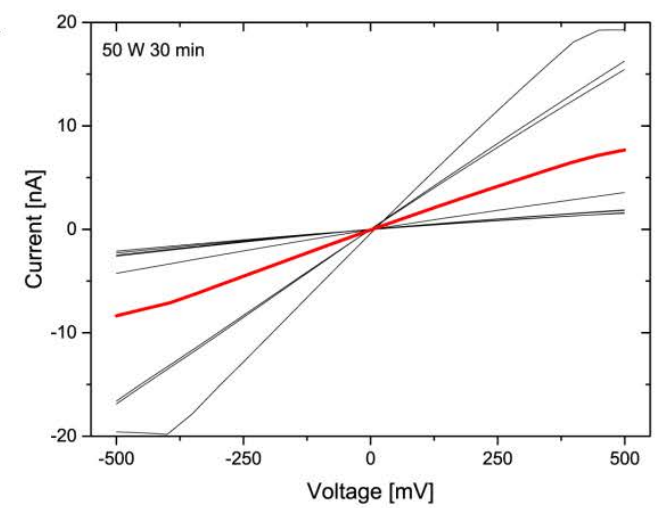

C

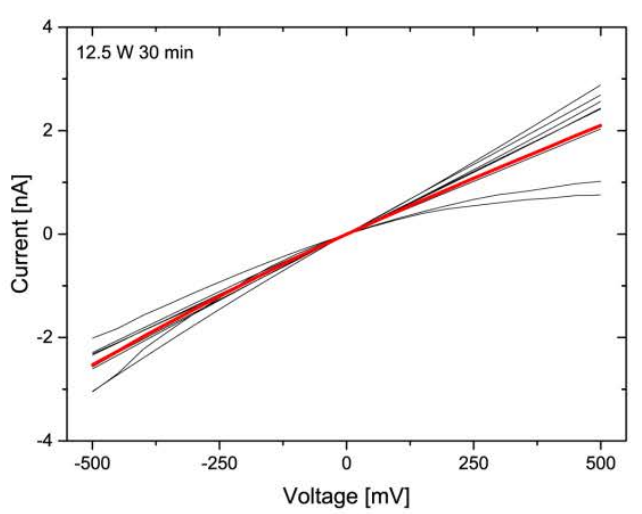

B

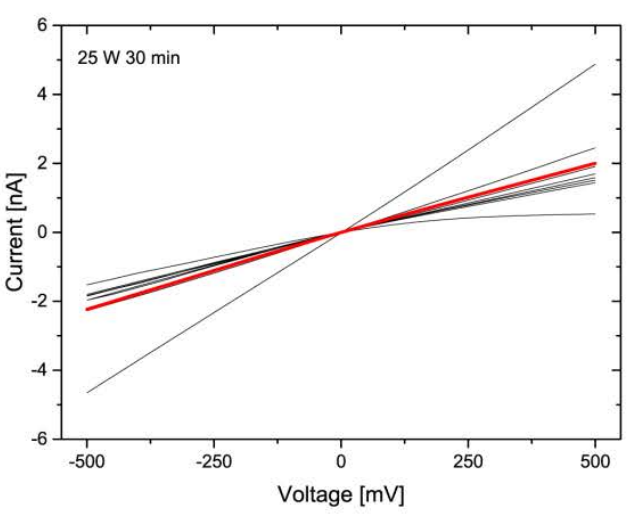

D

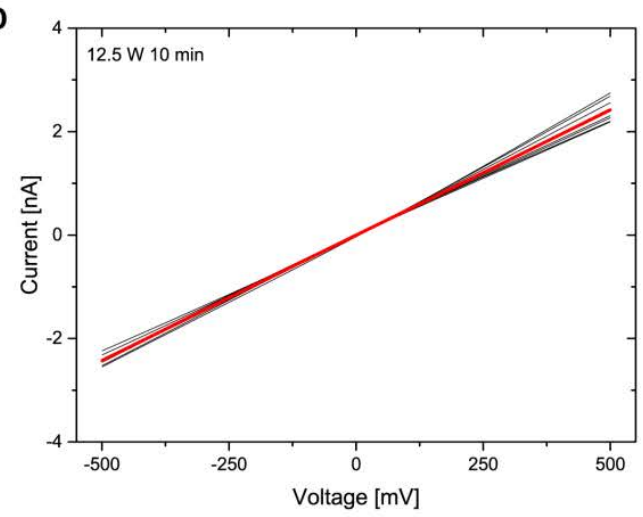

E

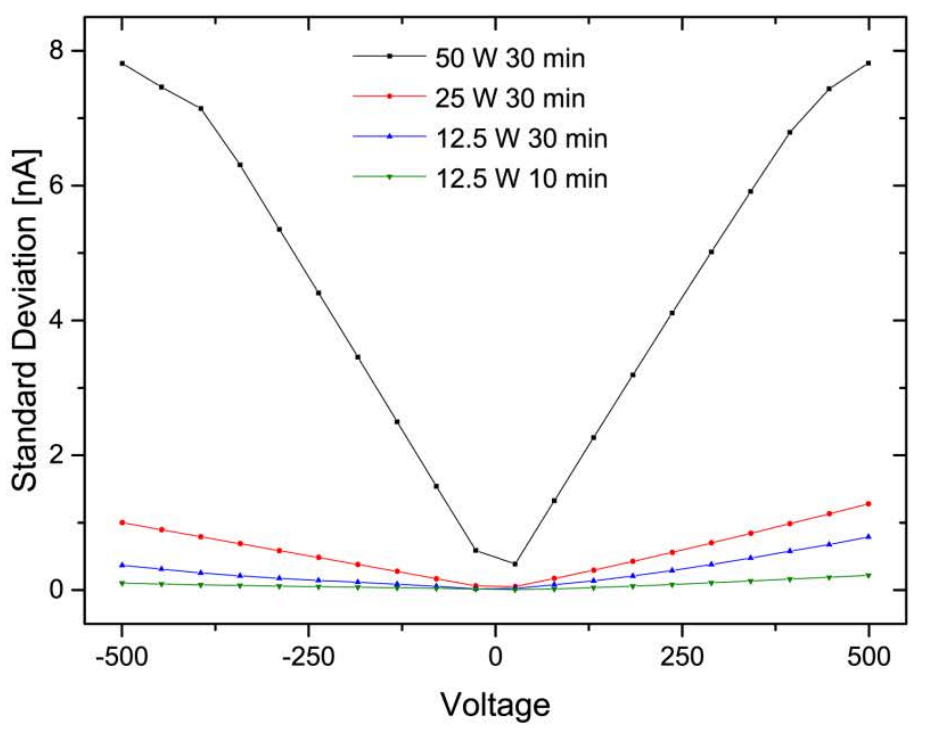

Figure S15. (A)-(D), I-V curves obtained after treatment with different $\mathrm{O}_{2}$-plasma powers and exposure times (13.56 MHz, $5 \mathrm{sccm}$ at 0.3 mbar). Each panel shows 8 GNCs pulled with the same program (black lines) and their average curve (red line). (E), Standard deviation comparison from each average curve of panels (A)-(D) reveals a significant influence of $\mathrm{O}_{2}$-plasma on the integrity of the GNCs during ion current measurements. 


\section{Single $I-V$ curves}

A moderate plasma procedure $(12.5 \mathrm{~W}$ for $10 \mathrm{~min})$ to facilitate wetting was applied to GNCs of programs 1 to 7 following which at least $50 \%$ of the GNCs were successfully wetted and show reproducible IV response. Clear outliers and damaged GNCs due to handling and/or the wetting procedure are not considered.
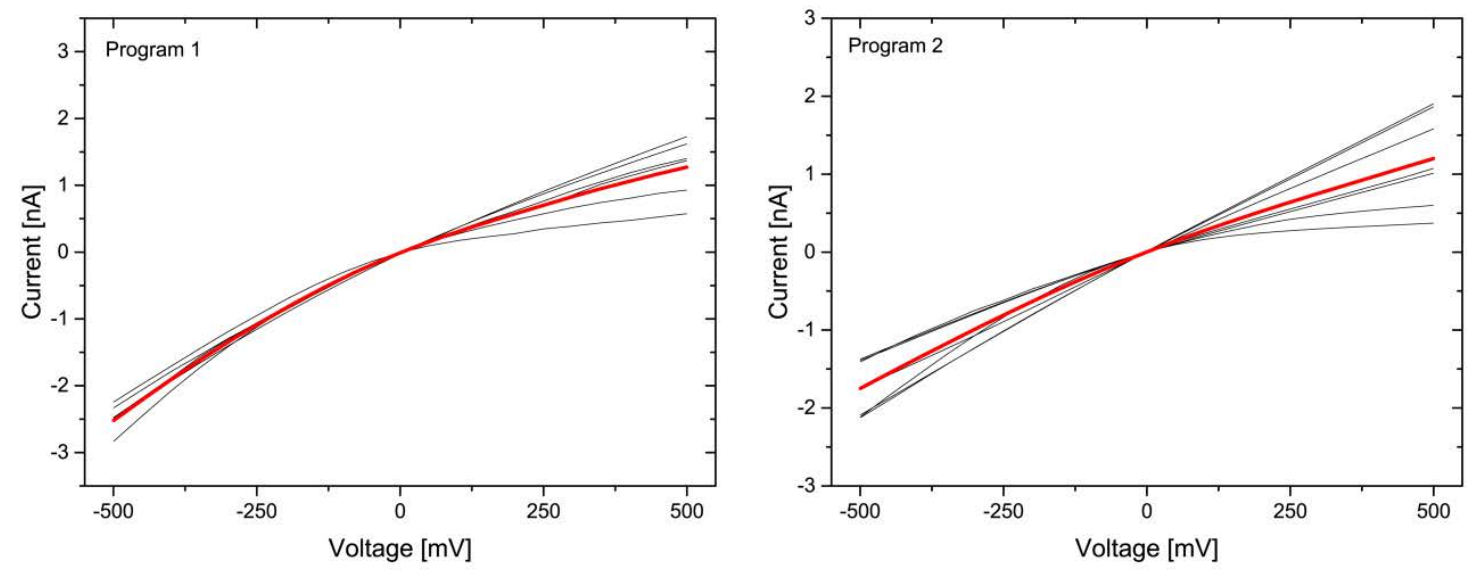

Figure S16. Single IV curves (black lines) and their average curve (red line) for bullet-like GNC tips from program $1(\mathrm{~N}=8)$ and program $2(\mathrm{~N}=7)$.
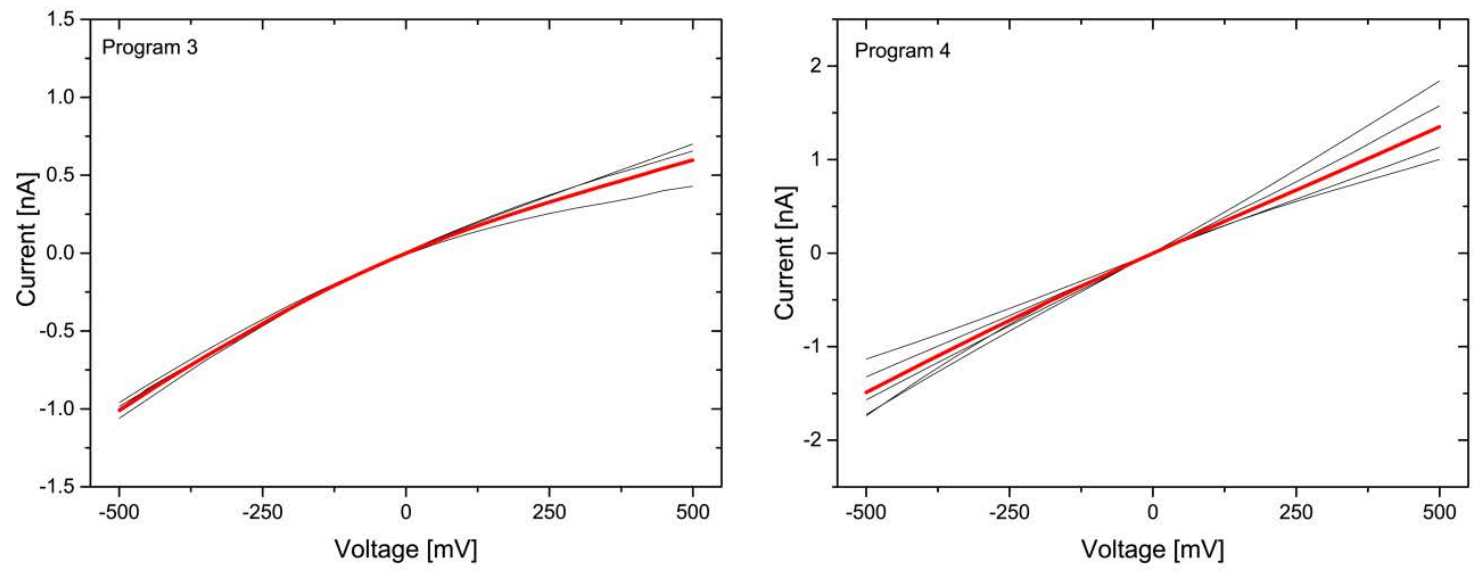

Figure S17. Single IV curves (black lines) and their average curve (red line) for blunt-ended GNC tips from program $3(\mathrm{~N}=6)$ and program $4(\mathrm{~N}=5)$. 

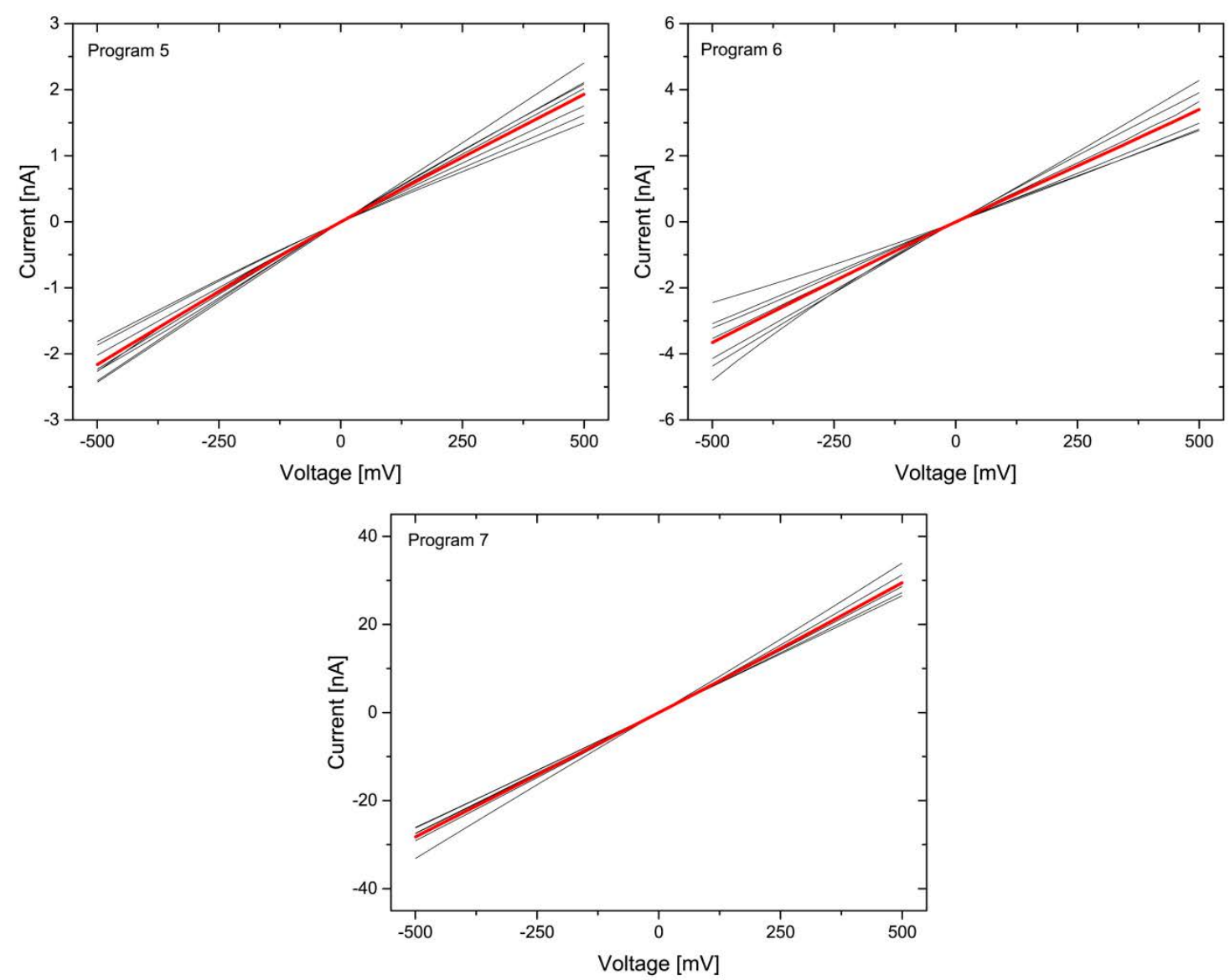

Figure S18. Single IV curves (black lines) and their average curve (red line) for hourglass shaped GNC tips from program $5(\mathrm{~N}=8)$, program $6(\mathrm{~N}=7)$ and program $7(\mathrm{~N}=8)$.

\section{References}

1. Li, W.; Bell, N. A.; Hernandez-Ainsa, S.; Thacker, V. V.; Thackray, A. M.; Bujdoso, R.; Keyser, U. F. Single Protein Molecule Detection by Glass Nanopores. ACS Nano 2013, 7, 4129-4134.

2. Steinbock, L. J.; Krishnan, S.; Bulushev, R. D.; Borgeaud, S.; Blokesch, M.; Feletti, L.; Radenovic, A. Probing the Size of Proteins with Glass Nanopores. Nanoscale 2014, 6, 14380-14387. 